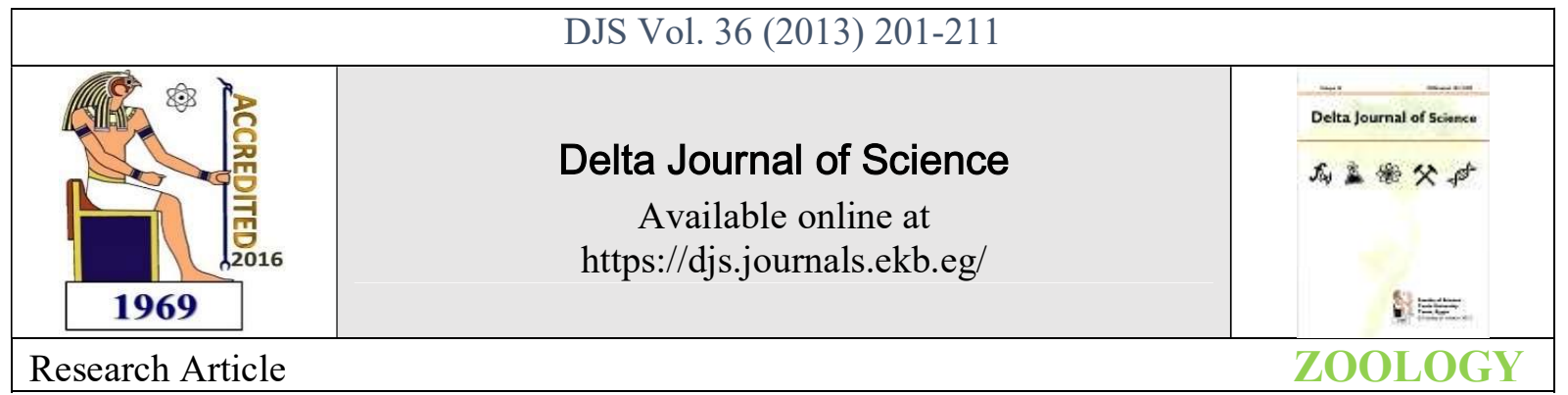

\title{
Recombinant expression of the rhoptry protein Clag3.1 as a green fluorescent chimeric protein in Plasmodium falciparum
}

\author{
Ahmed M. Ghoneim \\ Zoology Department, Faculty of Science, Damietta University, New Damietta, Egypt.

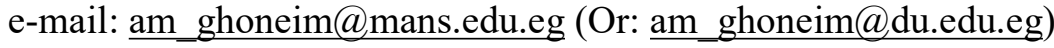 \\ Tel. ++20572403981, Fax. ++20572403868.
}

\begin{abstract}
Like all members of Apicomplexa, the motile form of Plasmodium, the merozoite, possesses an elaborate apical complex composed of 3 organelles; micronemes, rhoptries and dense granules. Invasion of erythrocytes by Plasmodium merozoites is a tightly regulated multi-step process that begins with the cooperation of proteins released from micronemes and rhoptries to mediate attachment of parasites to the host cell membrane and to establish the parasitophorous vacuole. RhopH complex is a very abundant rhoptry secretion composed of 3 protein members; RhopH1, RhopH2 and RhopH3. RhopH1 is represented by either Clag2, Clag3.1, or Clag9. RhopH2 was found to traffic to the rhoptries via its first 24 amino acids. To further address how the other members of RhopH complex proteins are trafficked to rhoptries, the present study established a new transgenic Plasmodium falciparum line expressing a large chimeric protein containing almost $3 / 4$ of Clag3.1 fused to the N-terminus of GFP via a linker region under the control of rhoph 2 promoter and its signal peptide encoding sequence. The data showed that this chimera was expressed efficiently indicating the strength of rhoph 2 promoter, however, the chimera was trapped in the early secretory pathway and did not reach rhoptries. Provided a correct folding of this protein, these data could imply that the middle part of Clag3.1 (aa 484-1064) contain some information that interfere with the targeting contained in the first 24 amino acids of RhopH2. The C-terminus of Clag3.1 could contain Clag3.1-specific rhoptry targeting information. Based on literature available in the international databases, this is the first report to successfully express such a large rhoptry chimeric GFP protein.
\end{abstract}

Key words: green fluorescent protein, Clag3.1, chimeric proteins, targeting, rhoptry, Plasmodium.

\section{INTRODUCTION}

Apicomplexan parasites, including the human pathogens Toxoplasma gondii and the malaria parasite Plasmodium falciparum, represent a phylum of protozoan parasites that are exclusively obligate intracellular parasites and must invade their host cells at some point in their lifecycle to complete development. All members of Apicomplexa are characterized by the presence, in the motile parasite form, of an apical complex composed of 3 secretory organelles; micronemes, rhoptries, and dense granules; the contents of which are discharged sequentially upon invasion and are crucial for parasite invasion of the host cell and formation of the parasitophorous vacuole (Bradley and Sibley, 2007; Boothroyd and Dubremetz, 2008; Tufet-Bayona et al., 2009).
After intimate contact of the parasite and the host cell, secretion of rhoptry proteins is initiated very rapidly and completed within a few minutes of invasion, implying that the contents of rhoptries are vital in the establishment of the parasitophorous vacuole (Dowse and Soldati, 2005; Bradley and Sibley, 2007; Kaneko, 2007; Kats et al., 2008; TufetBayona et al., 2009). Among proteins identified in the rhoptries of Plasmodium merozoites is the high molecular mass RhopH complex which is a heterodimer composed of

three proteins: RhopH1, RhopH2, and RhopH3 (Campbell et al., 1984; Holder et al., 1985; Cooper et al., 1988; Hienne et al., 1998). RhopH1 is encoded by three members of the rhoph1/clag family, namely, clag2, clag3.1, and clag9 (Kaneko et al., 2001; Ling et al., 2004; Kaneko et al., 2005). 
Although thought to be involved in erythrocyte binding during or after invasion (Sam-Yellowe, 1992; Rungruang et al., 2005) and participating in the formation of the parasitophorous vacuolar membrane (Ling et al., 2003; 2004), the exact function of the complex has not been determined yet.

In mammalian cells, sorting of transmembrane proteins is mediated by cytoplasmic adaptor complexes (APs) which recognize specific motifs (e.g. the YXXø motif, where $\varnothing$ is a hydrophobic amino acid) within their cytoplasmic tails. APs select cargo for inclusion into a transport vesicle and recruit coat components (e.g. clathrin) necessary for vesicular budding and transport (Robinson, 2004; Bonifacino and Lippincott-Schwartz J., 2003). This mechanism has been shown to operate in Toxoplasma, and may also be conserved in Plasmodium (Hoppe et al., 2000; Bhanot et al., 2003). However, most Plasmodium rhoptry proteins described to date do not possess a transmembrane region and cytoplasmic tail, implying the existence of additional sorting pathways (Kats et al., 2006). One possibility is that sorting within the Golgi occurs via a clustering mechanism whereby proteins en route to a particular destination aggregate into distinct sub-domains like what happens in mammalian cells (Glombik and Gerdes, 2000).

Proteins targeted to the apicoplast share general features and use common targeting signals (Foth et al., 2003) and so the proteins are exported to the host erythrocyte (Hiller et al., 2004; Marti et al., 2004). However, rhoptry proteins do not have a yet identified common signal to target them to their destination. Since rhoptry proteins are important in the formation of the parasitophorous vacuoles and in the invasion of host cell, the accurate targeting of these proteins to their final destination must be an essential process for the invasion of erythrocytes and the growth of parasites.

The rhoptry associated membrane antigen (RAMA) is a glycosylphosphatidyl inositol-anchored protein that is synthesized and expressed first in the late ring stage before the appearance of recognizable rhoptries, and appears to temporarily accumulate within compartments of the secretory pathway (Topolska et al., 2004). Fluorescence Resonance Energy Transfer experiments indicated that RAMA interacts with the low molecular weight (LMW) rhoptry complex (Topolska et al., 2004). Rhoptry targeting of the LMW complex (heterodimer composed of RAP1, and RAP2 or RAP3) occurs via the N-terminus of RAP1, although the mechanism is not understood (Baldi et al., 2000). RAMA has been hypothesized to act as an escorter for RAP1 to recruit RAP1, RAP-2 and RAP-3 into a rhoptry-destined protein complex.

Ghoneim et al. (2007) determined that the N-terminal 24 amino acids of RhopH2, including signal peptide sequence, are sufficient to target GFP to the rhoptries under the control of rhoph 2 promoter and proposed that this targeting is likely mediated by a unique mechanism that depends on the interaction with $\mathrm{N}$-terminal 24 amino acids of RhopH2 early in the secretory pathway. This targeting has been shown to be sensitive to Brefeldin A and thus implied that the 5 amino acids downstream of the signal peptide cleavage site may contain the sorting signal required for rhoptry targeting (Ghoneim, in press). The present study tried to determine whether Clag3.1, a member of RhopH complex, is trafficked to rhoptries under the same control elements or rather uses an alternative targeting mechanism. To do this, a new transgenic Plasmodium falciparum line expressing $3 / 4$ of Clag 3.1 fused to the N-terminus of GFP under the control of RhopH2 promoter was established and the expression the GFP chimera was monitored in live parasites.

\section{Materials and Methods}

1. Construction of the plasmid expressing the $\mathrm{N}$-terminal $3 / 4$ of Clag3.1 as GFP chimeric protein

The TCGCGGATCCTATGTGACATCACTTTATTTACCAGG and the reverse primer TCGCGGATCCACATATCATACATTTTGGATGCTAGC were used to amplify a region encoding the middle 570 amino acids of Clag3.1 (amino acids (aa) 461-1030) from a plasmid clone containing a part of clag3.1 coding sequence (HB3 strain). Amplicons were subcloned, sequenced and the BamHI restriction fragment was ligated into the BglII site that exists at $3^{\prime}$ end of clag3.1 in the plasmid pRGDT-Clag3.1A-B12 (Ghoneim et al., 2007). This step produced the plasmid pRGDT-Clag3.1AB-B12 (Fig. 1). Size of this plasmid was confirmed by restriction digestion with NheI and SpeI enzymes (Fig. 2). The plasmid was then subjected to BP Gateway recombination reaction with the donor vector pDONR ${ }^{\mathrm{TM}} 221$ (Invitrogen) according to the manufacturer's instructions to produce the corresponding entry clones pENTR.RGDT-Clag3.1AB (Fig. 1). Size of this plasmid was confirmed by restriction digestion with NheI and EcoRV enzymes (Fig. 2). The plasmid was then subjected to LR Gateway recombination reaction according to Invitrogen instructions with $\mathrm{pH} . \mathrm{DEST} / \mathrm{H}$ (Ghoneim et al., 2007) to produce the final stable transfection vector pH.RGDTClag3.1AB (Fig. 1). Size of this plasmid was confirmed by restriction digestion with EcoRI and EcoRV enzymes (Fig. 2). The multiple cloning site before GFP was removed by restricting this plasmid at the endogenous NheI site (at $3^{\prime}$ the end of the cloned part of clag3.1) and NheI of the multiple cloning site. The cohesive ends were filled in and self ligated to recreate NheI site again.

To provide flexibility to the hydrocarbon chain before GFP, we added a Poly-Glycine linker into pH.RGDT-Clag3.1AB as follows: The two oligos CTAGAGGAGGCGGGGGTAGCGGTGGAGGCTCCGGG GGTGGAGGGCTCGAGT and CTAGACTCGAGCCCTCCACCCCCGGAGCCTCCACCG

CTACCCCCGCCTCCT (with XhoI sites underlined) were annealed and ligated into the recreated NheI (that exists at $3^{\prime}$ the end of the cloned part of Clag3.1) in the construct pH.RGDT-Clag3.1AB to produce the construct $\mathrm{pH}$.RGDTClag3.1AB-Glink which expresses the N-terminal $3 / 4$ of the full length of PfClag3.1 (aa 24-1030) as a chimeric protein fused with c-Myc to its $\mathrm{N}$-terminus and GFP to its $\mathrm{C}$-terminus.

\section{DNA sequence Analysis}

Plasmids were sequenced at different steps of cloning to select the desired clones and to determine whether any mutation has been created. The linker region was sequenced to confirm its correct direction. In all steps, plasmid DNA was amplified with $0.5 \mu 1$ of Big Dye Terminator (Applied Biosystems), 1.2 pmol of primer and $1.25 \mu 1$ of $5 \times$ Big Dye sequencing buffer according to Applied Biosystems' instructions. Products were precipitated with $4 \mu \mathrm{l}$ of $3 \mathrm{M}$ Sodium Acetate and $100 \mu \mathrm{l}$ of 95\% Ethanol according to the standard protocols. At the end, $20 \mu \mathrm{Hi}$-Dye formamide were mixed with the DNA, heated for 2 min at $95^{\circ} \mathrm{C}$ and chilled on ice and finally loaded to ABI PRISM 3100 DNA sequence.

\section{Parasite culture and transfection}

The P. falciparum (Dd2 strain) was grown in vitro according to the standard protocol (Trager and Jensen, 1976) with little modifications as previously described (Ghoneim, 2009). Parasite transfection was carried out according to Deitsch et al. (2001) by loading erythrocytes with $100 \mu \mathrm{g}$ of the transfection construct pH.RGDT-Clag3.1AB-Glink by 
electroporation under the previously mentioned conditions (Ghoneim, 2009). These plasmid-loaded erythrocytes were inoculated by late stage parasites, allowed to be invaded and incubated for 3 days. Transfected parasites were selected by applying the drug WR99210 at $10 \mathrm{nM}$ concentration and the culture was monitored by investigating Giemsa-stained blood smears every 2 days.

\section{Parasite synchronization}

To synchronize parasite cultures, infected erythrocytes were collected by centrifugation and resuspended in $2 \mathrm{ml}$ complete medium. 10× volume of $5 \%$ Sorbitol were added and the suspension was incubated at $37^{\circ} \mathrm{C}$ for $8 \mathrm{~min}$ (Lambros and Vanderberg, 1979). Intact erythrocytes were collected by centrifugation and the Sorbitol solution was immediately removed. Parasite pellet was washed once with $10 \mathrm{ml}$ of incomplete medium and resuspended in complete medium. The same parasite culture was synchronized once more 26 hours later and then put back into the culture media and grown for one cycle before investigation. Representative images of synchronized cultures are shown in Fig. 2.

\section{Microscopy}

Small aliquots of parasite culture were washed and incubated with PBS solution containing the nuclear stain 4', 6diamidino-2-phenylindole (DAPI) for $5 \mathrm{~min}$. Parasites were mounted without further treatment and GFP fluorescent parasites were imaged using a fluorescence microscope connected to a digital camera. Images were processed using Adobe Photoshop software.

\section{Western blot analysis}

Proteins were extracted as described before (Ghoneim et al., 2007) from the parasites expressing Clag3.1-GFP recombinant protein and from non-transfected parasites. Briefly, proteins were extracted by repeated freeze-thaw cycles, dissolved in $1 \times$ SDS-PAGE loading buffer, incubated at $100{ }^{\circ} \mathrm{C}$ for $3 \mathrm{~min}$ and run on $5-20 \%$ polyacrylamide gel. Proteins were blotted onto PVDF membrane (Bio-Rad) and immunostained with the specific mouse anti-Myc monoclonal antibody followed by staining with horseradish peroxidase-conjugated secondary antibody. Stained membranes were visualized with ECL Plus (Amersham, Biosciences) on a sensitive film.

\section{RESULTS}

\section{Description of the final transfection vector and the recombinant Clag3.1}

To monitor the trafficking of Clag3.1, the present study constructed a large transfection vector $\mathrm{pH}$.RGDT-Clag3.1ABGlink to expresses $3 / 4$ of Clag3.1 fused to the RhopH2 signal peptide encoding sequence and under the control of the rhoptry gene pfrhoph2 promoter. This vector has some advantages that facilitate manipulating it to express any protein of interest under the desired conditions in case of absence of the recombination reagents. The vector is characterized by the presence of unique restriction enzyme sites ClaI and SpeI that permit changing the pfrhoph2 promoter and the signal peptide-encoding sequence. Sall site after GFP permits adding new sequences downstream to GFP if desired to express it within the protein of interest. GFP itself can be replaced by another fluorescent tag or removed from this final construct easily by digesting it with $\mathrm{XbaI}$ and SalI enzymes.
The sequence encoding Clag3.1 in the transfection vector was sequenced at the different stages of recombination reactions and translated by the ExPASy tool (Bioinformatics Resource Portal). This chimera, as shown in Fig. 3, would be composed of 1324 amino acids and contain a c-Myc tag at the Nterminus just 7 amino acids downstream to the signal peptide cleavage site. The chimera would contain a C-terminal GFP tag composed of 238 amino acids. Between the carboxylic terminus of Clag. 3 and the N-terminus of GFP there would be a Glycine-rich linker region composed of 11 glycine residues with interspersed 2 Serine residues. Also, this chimeric protein would contain 10 cysteine residues, the first 9 of which are conserved throughout the RhopH1/Clag family and the last one is a partially conserved residue.

\section{Tracking the expression of RhopH2-Myc-Clag3.1(24- 1064)-GFP chimeric protein}

Ring and trophozoite stages of the transfected parasites did not show any fluorescence signal but a localized green signal was detected in schizont stage parasites. In the two nuclei-stage schizonts, one or two dot-like green fluorescent signals were detected in the parasite cytoplasm. No fluorescence signal was observed at the periphery of the parasite (Fig. 4 A). With the growth of parasites to 3, 4 and 5 nuclei-stage schizonts (Fig. 4 $\mathrm{B}, \mathrm{C}, \mathrm{D})$, the pattern of fluorescence signal remained dot-like and localized within the parasite cytoplasm. The number of these dot-like signals increased with the parasite growth and no fluorescence was seen neither distributed in the whole cytoplasmic area nor at the periphery of the parasites. This fluorescence pattern is consistent with the localization of the GFP chimeric protein in the parasite cytoplasm. This protein did not reach the parasitophorous vacuole but instead was retained to a specific compartment in the cytoplasm that is most likely to be a specific location in the endoplasmic reticulum or within Golgi of the parasite. The green fluorescence signal could not be detected with further parasite development to the mature schizonts or in the released merozoites which confirms the inability of this chimera to be targeted to the rhoptries as a final destination.

\section{Detection of RhopH2-Myc-Clag3.1(24-1064)-GFP chimeric protein by Western blot}

To verify the integrity of the expressed chimeric protein, total proteins were extracted from the transfected parasites and analyzed by Western blot. As seen in Fig. 5, anti-C-Myc detected a specific band close to $150 \mathrm{kDa}$. This coincides with the expected molecular weight $(145.5 \mathrm{kDa})$ of the expressed RhopH2-Myc-Clag3.1(24-1064)-GFP protein and confirms the expression of the complete RhopH2-Myc-Clag3.1(24 1064)-GFP protein without any truncation. Altogether, the data show the successful expression of the Clag3.1-GFP chimera and indicate that the rhoph2 promoter is a very strong promoter that is reliable in driving the expression of large chimeric proteins.

\section{Discussion}

At least 12 subcellular and extracellular destinations have been identified for secretory proteins in Plasmodium parasites (Van Dooren et al., 2000). Trafficking of proteins to most cellular destinations requires specific targeting or retention signals. Targeting motifs for some of these destinations have been revealed while others still remain elusive.

Ghoneim et al. (2007) determined that the N-terminal 24 amino acids of RhopH2, including signal peptide sequence, are sufficient to target GFP to the rhoptries under the control of rhoph 2 promoter. To further address whether some internal 
motifs or domains control trafficking of the soluble rhoptry proteins to the rhoptry organelles of Plasmodium falciparum, the present study constructed a large plasmid vector that would express almost $3 / 4$ of Clag3.1 (aa 24-1064) tagged with GFP at the C-terminus.

The establishment of large transfection vector like pH.RGDTClag3.1AB-Glink (10732 bp) by conventional and standard cloning techniques is considered a challenging process for the long time needed to achieve this and the difficulty to clone the Plasmodium AT rich sequences $(80 \% \mathrm{~A} / \mathrm{T}$, relative to $60 \%$ in human DNA) which leads to deletions and rearrangements in the resulting clones (Gardner et al., 2002). The current strategy facilitated a little bit this process through constructing the transfection vector in two steps; the first includes producing an entry clone carrying the gene of interest cassette. The second includes recombining this entry clone with a destination vector carrying the drug resistance gene cassette to produce the final transfection vector. Ghoneim et al. ( 2007) used the same strategy and successfully produced smaller transfection vectors that were introduced to Plasmodium falciparum and successfully expressed GFP chimeric proteins that were targeted efficiently to the rhoptries. In the same study another large transfection vector encoding the full length of Clag3.1 was produced but, unfortunately, the complete GFP fusion protein could not be expressed in Plasmodium parasites due to chopping of the GFP from the chimera in addition to the loss of around 390 amino acids from the C-terminus of Clag3.1 most probably due to misfolding. The authors thought that the truncation of the chimeric protein and loss of GFP could be due to the inflexible link between GFP and Clag3.1. Therefore, in the current study, a flexible Glycine-rich linker was designed and used to produce $3 / 4$ of Clag3.1 fused to $\mathrm{N}$ terminus of GFP through this linker. The data showed that the GFP fusion protein could be expressed successfully and localized to the parasite cytoplasm. The chimera was trapped in a specific compartment in the cytoplasm that is most likely to be the endoplasmic reticulum or Golgi of the parasite and was never detected in the parasitophorous vacuole of the parasite.

Rhoptry proteins are well known to pass through the ER and Golgi (Howard and Schmidt, 1995; Noe et al., 2000; Ghoneim, in press), therefore, it is likely that this chimera was arrested in the ER or Golgi. One potential reason could be the improper folding of this large chimeric protein. In fact, this expressed chimeric protein misses the most $\mathrm{C}$-terminal 4 Cysteine residues existing in the endogenous Clag3.1 which could impart specific folding features for Clag3.1. Insertion of GFP in the far C-terminus of the large protein and in place of Cysteine residues could disturb the folding of Clag3.1. According to Striepen et al. (2001), several MIC3-GFP deletion mutants appeared to arrest in the secretory pathway, in compartments thought to be either ER or Golgi, probably due to improper folding because of the high Cysteine content of TgMIC3. Retention of improperly folded proteins in the ER-Golgi by chaperone complexes is a well documented quality control step in other eukaryotic systems (Ellgaard et al., 1999). The GFP tag, as a relatively large moiety, may potentially interfere with the native structure and function of the target protein (Yu et al., 2003).

It is noteworthy to mention that successful GFP fluorescence indicates that GFP itself has folded correctly and this may imply that the expressed Clag 3.1 could have folded correctly too. By expressing the protein of interest fused to GFP in $E$. coli and measuring fluorescence, March et al. (2003) found that folding of the GFP chromophore was an accurate indicator of target protein-folding. Analysis of many research articles revealed that most of the fusion proteins have the target proteins connected to the N-terminus of GFP without using linker sequences (Yu et al., 2003) and GFP fusion at the $\mathrm{C}$-terminal end of target proteins does not interfere with target protein function and localization (Lee et al., 2001; Chen et al., 2000). Strategically, the current study added a Serinecontaining Glycine linker between GFP and Clag3.1 to avoid truncating GFP and any possible interference with the folding of Clag3.1. With Glycine residues enhancing solubility and Serine providing little rigidity to the polypeptide chain, many studies have demonstrated that the addition of a flexible Glycine/Serine linker between the two parts of a fusion protein allows the expression of a fusion protein retaining functional activity in a variety of host systems $E$. coli, yeast and humans (Guillet et al., 2002; Anderson et al.; 2010). If this is the case and the RhopH2-Myc-Clag3.1(24-1064)-GFP has folded correctly, it remains to explain why this chimera has been retained in the early secretory pathway. One possibility is that the middle part of Clag3.1 (aa 484-1064) contains some information that intervene with the targeting information contained in the first 24 amino acids of RhopH2 which are sufficient to target GFP to rhoptries (Ghoneim et al., 2007). Alternatively, the C-terminus of Clag3.1 (missing from this chimera) could contain the rhoptry targeting information specific for Clag3.1. In the closely related Apicomplexan parasite T.gondii, there are controversial reports about the signals necessary for targeting rhoptry proteins to their destinations. The rhoptry protein TgROP 1 was successfully targeted to the rhoptries with either the propeptide region (aa 1-85) or a central fragment composed of 372 amino acid (Striepen et al., 2001). Moreover, targeting of rhoptry protein TgROP4 was achieved by either its C-terminal Tyr-based motif (YXXø) (Hoppe et al., 2000) or by the N-terminal 163 amino acids (Bradley et al., 2004).

Previously the author and others have shown that the middle third of Clag3.1 is necessary for Clag3.1 to associate the RhopH complex. Clag3.1 portion expressed in the current study contains 4 Cysteine residues conserved among RhopH1/Clag family and whether they have a role in protein trafficking has to be clarified. Provided correct folding of the chimera produced in this study, it seems that these 4 Cysteine residues are not enough to target proteins to the rhoptries and consequently that the C-terminus of Clag 3.1 could contain the rhoptry targeting information specific for Clag3.1. This conclusion is supported negatively by the results of Bioinformatic analysis of middle region of Clag 3.1 which did not reveal the presence of any specific motifs or domains.

Identification of the specific motifs and or domains responsible for targeting rhoptry proteins could help in designing therapeutic drugs, if not vaccines, to interfere with the invasion of erythrocytes by Plasmodium and hence combating malaria. Based on the information available in the international databases, this GFP chimeric rhoptry protein is the largest chimera produced in Plasmodium.

\section{Conclusion}

In this study, $3 / 4$ of Clag3.1 was successfully expressed as GFP chimeric protein and GFP signal was strong enough for detection after the relatively large Clag3.1. This validates the rhoph 2 promoter for driving expression of large chimeras and also proves that the glycine-rich linker has provided flexibility for GFP and prevented its truncation. Clag3.1-GFP chimera was retained in a specific cytoplasmic compartment; most likely endoplasmic reticulum or Golgi of the parasite. Provided correct folding of the chimera, theses data could imply alternative role for the C-terminus of Clag3.1 in protein targeting. In general, the strategy outlined here can be 
Ahmed M. Ghoneim., Recombinant expression of the rhoptry protein Clag3.1 as a green fluorescent chimeric protein in Plasmodium falciparum.

exploited in the expression of other biological molecules in many other biological systems.

\section{Acknowledgements}

Author would like to thank professor Motomi Torii, Department of Molecular Parasitology, Ehime University School of Medicine (Japan) and professor Osamo Kaneko, Department of Protozoology, Institute of Tropical Medicine, Nagasaki University (Japan) for their help and guidance during his stay in Japan

\section{References}

Anderson J.C.; Dueber J.E.; Leguia M.; Wu G.C.; Goler J.A.; Arkin A.P.; Keasling J.D. (2010): BglBricks: A flexible standard for biological part assembly. J. Biol. Eng.; 4: 1.

Baldi DL; Andrews KT; Waller RF; Roos DS; Howard RF; Crabb BS; and Cowman AF (2000): RAP1 controls rhoptry targeting of RAP2 in the malaria parasite Plasmodium falciparum. EMBO J.; 19: 2435-2443.

Bhanot P.; Frevert U.; Nussenzweig V.; Persson C. (2003): Defective sorting of the thrombospondin-related anonymous protein (TRAP) inhibits Plasmodium infectivity. Mol. Biochem. Parasitol.; 126: 263-273.

Bonifacino J.S.; Lippincott-Schwartz J. (2003): Coat proteins: shaping membrane transport. Nat. Rev. Mol. Cell Biol.; 4: 409-414.

Boothroyd J.C.; Dubremetz J.F. (2008): Kiss and spit: the dual roles of Toxoplasma rhoptries. Nat. Rev. Microbiol.; 6:79-88.

Bradley P.J.; Li N.; Boothroyd J.C. (2004): A GFP-based motif-trap reveals a novel mechanism of targeting for the Toxoplasma ROP4 protein. Mol. Biochem. Parasitol.; 137:111-20.

Bradley P.J.; Sibley L.D. (2007): Rhoptries: an arsenal of secreted virulence factors. Curr. Opin. Microbiol.; 10: 582 587.

Campbell G.H.; Miller L.H.; Hudson D.; Franco E.L.; Andrysiak P.M. (1984): Monoclonal antibody characterization of Plasmodium falciparum antigens. Am. J. Trop. Med. Hyg.; 33: $1051-1054$

Chen B.; Harms E.; Chu T.; Henrion G.; Strickland S. (2000): Completion of meiosis in Drosophila oocytes requires transcriptional control by grauzone; a new zinc finger protein. Development; 127: 1243-1251.

Cooper J.A.; Ingram L.T.; Bushell G.R.; Fardoulys C.A.; Stenzel D.; Schofield L.; Saul A.J. (1988): The 140/130/105 kilodalton protein complex in the rhoptries of Plasmodium falciparum consists of discrete polypeptides. Mol. Biochem. Parasitol.; 29: 251-260.

Deitsch K.; Driskill C. and Wellems T. (2001): Transformation of malaria parasites by the spontaneous uptake and expression of DNA from human erythrocytes. Nucleic Acids Res.; 29: 850-853.

Dowse T.J.; Soldati D. (2005): Rhomboid-like proteins in Apicomplexa: phylogeny and nomenclature. Trends Parasitol.; 21: 254-258

Ellgaard L.; Molinari M.; Helenius A. (1999): Setting the standards: quality control in the secretory pathway. Science; 286: $1882-1888$.

Foth B.J.; Ralph S.A.; Tonkin C.J.; Struck N.S.; Fraunholz M.; Roos D.S.; Cowman A.F.; McFadden G.I. (2003):
Dissecting apicoplast targeting in the malaria parasite Plasmodium falciparum. Science; 299: 705-708.

Gardner M.J.; Hall N.; Fung E.; White O.; Berriman M.; Hyman R.W.; Carlton J.M.; Pain A.; Nelson K.E.; Bowman S.; Paulsen I.T.; James K.; Eisen J.A.; Rutherford K.; Salzberg S.L.; Craig A.; Kyes S.; Chan M.S.; Nene V.; Shallom S.J.; Suh B.; Peterson J.; Angiuoli S.; Pertea M.; Alien J.; Selengut J.; Haft D.; Mather M.W.; Vaidya A.B.; Martin D.M.; Fairlamb A.H.; Fraunholz M.J.; Roos D.S.; Ralph S.A.; McFadden G.I.; Cummings L.M.; Subramanian G.M.; Mungall C.; Venter J.C.; Carucci D.J.; Hoffman S.L.; Newbold C.; Davis R.W.; Fraser C.M.; Barrell B. (2002): Genome sequence of the human malaria parasite Plasmodium falciparum. Nature; 419:498-511.

Ghoneim A.; Kaneko O.; Tsuboi T.; Torii M. (2007): The Plasmodium falciparum RhopH2 promoter and first 24 amino acids are sufficient to target proteins to the rhoptries. Parasitol. Int.; 56: 31-43.

Ghoneim A.M. (2009): Cloning and characterization of some rep20 DNA fragments from the genome of the human malaria pathogen Plasmodium falciparum. J. Egypt Soc. Parasitol.; 39: 489-502.

Ghoneim; A.M. (in press): Trafficking of Plasmodium falciparum chimeric rhoptry protein with Brefeldin A. Folia Parasitologica http://folia.paru.cas.cz/detail.php?id=22103).

(Online:

Glombik M.M.; Gerdes H.H. (2000): Signal-mediated sorting of neuropeptides and prohormones: secretory granule biogenesis revisited. Biochimie.; 82: 315-326.

Guillet C.; Lelièvre E.; Plun-Favreau H.; Froger J.; Chabbert M.; Hermann J.; Benoit D.E.; Coignac A.; Bonnefoy J.Y.; Gascan H.; Gauchat J.F.; Elson G. (2002): Functionally active fusion protein of the novel composite cytokine CLC/soluble CNTF receptor. Eur. J. Biochem.; 269: 1932-1941.

Hienne R.; Ricard G.; Fusaï T.; Fujioka H.; Pradines B.; Aikawa M.; Doury J.C.(1998): Plasmodium yoelii: identification of rhoptry proteins using monoclonal antibodies. Exp. Parasitol.; 90: 230-235.

Hiller N.L.; Bhattacharjee S.; van Ooij C.; Liolios K.; Harrison T.; Lopez-Estraño C.; Haldar K. (2004): A hosttargeting signal in virulence proteins reveals a secretome in malarial infection. Science; 306: 1934-1937.

Holder A.A.; Freeman R.R.; Uni S.; Aikawa M. (1985) Isolation of a Plasmodium falciparum rhoptry protein. Mol. Biochem. Parasitol.; 14: 293-303.

Hoppe H.C.; Ngo H.M.; Yang M.; Joiner K.A. (2000): Targeting to rhoptry organelles of Toxoplasma gondii involves evolutionarily conserved mechanisms. Nat. Cell. Biol.; 2: 449-456.

Howard R.F.; Schmidt C.M. (1995): The secretory pathway of Plasmodium falciparum regulates transport of p82/RAP1 to the rhoptries. Mol. Biochem. Parasitol.; 74: 43-54.

Kaneko O. (2007): Erythrocyte invasion: vocabulary and grammar of the Plasmodium rhoptry. Parasitol. Int.; 56: 255262.

Kaneko O.; Tsuboi T.; Ling I.T.; Howell S.; Shirano M.; Tachibana M.; Cao Y.M.; Holder A.A.; Torii M. (2001): The high molecular mass rhoptry protein; RhopH1; is coded by members of the clag multigene family in Plasmodium falciparum and Plasmodium yoelii. Mol. Biochem. Parasitol.; 118: 223-231. 
Kaneko O.; Yim Lim B.Y.; Iriko H.; Ling I.T.; Otsuki H.; Grainger M.; Tsuboi T.; Adams J.H.; Mattei D.; Holder A.A.; Torii M. (2005): Apical expression of three RhopH1/Clag proteins as components of the Plasmodium falciparum RhopH complex. Mol. Biochem. Parasitol.; 143: 20-28.

Kats L.M.; Black C.G.; Proellocks N.I.; Coppel R.L. (2006): Plasmodium rhoptries: how things went pear-shaped. Trends Parasitol.; 22: 269-276.

arrest specific 1 gene in the development of the mouse embryo. Dev. Biol.; 234: 188-203.

Ling I.T.; Florens L.; Dluzewski A.R.; Kaneko O.; Grainger M.; Yim Lim B.Y.; Tsuboi T.; Hopkins J.M.; Johnson J.R.; Torii M.; Bannister L.H.; Yates J.R. 3rd; Holder A.A.; Mattei D. (2004): The Plasmodium falciparum clag9 gene encodes a rhoptry protein that is transferred to the host erythrocyte upon invasion. Mol. Microbiol.; 52: 107-118.

Ling I.T.; Kaneko O.; Narum D.L.; Tsuboi T.; Howell S.; Taylor H.M.; Scott-Finnigan T.J.; Torii M.; Holder A.A. (2003): Characterisation of the rhoph2 gene of Plasmodium falciparum and Plasmodium yoelii. Mol. Biochem. Parasitol.; 127: $47-57$

March J.C.; Rao G. and Bentley W.E. (2003): Biotechnological applications of green fluorescent protein. Appl. Microbiol. Biotechnol.; 62: 303-315.

Marti M.; Good R.T.; Rug M.; Knuepfer E.; Cowman A. F. (2004): Targeting malaria virulence and remodeling proteins to the host erythrocyte. Science; 306: 1930-1933.

Noe A.R.; Fishkind D.J.; Adams J.H. (2000): Spatial and temporal dynamics of the secretory pathway during differentiation of the Plasmodium yoelii schizont. Mol. Biochem. Parasitol.; 108: 169-185.

Robinson M.S. (2004): Adaptable adaptors for coated vesicles. Trends Cell Biol.; 14: 167-174.

Rungruang T.; Kaneko O.; Murakami Y.; Tsuboi T.; Hamamoto H.; Akimitsu N.; Sekimizu K.; Kinoshita T.; Torii M. (2005): Erythrocyte surface glycosylphosphatidyl inositol anchored receptor for the malaria parasite. Mol. Biochem. Parasitol.; 140: 13-21.

Sam-Yellowe T. Y. (1992): Molecular factors responsible for host cell recognition and invasion in Plasmodium falciparum. J. Protozool.; 39: 181-189.

Striepen B.; Soldati D.; Garcia-Reguet N.; Dubremetz J.F.; Roos D.S. (2001): Targeting of soluble proteins to the rhoptries and micronemes in Toxoplasma gondii. Mol. Biochem. Parasitol.; 113: 45-53

Topolska A.E.; Lidgett A.; Truman D.; Fujioka H.; Coppel R.L. (2004): Characterization of a membrane-associated rhoptry protein of Plasmodium falciparum. J Biol Chem.; 279:4648-56

Trager W.; Jensen J.B. (1976): Human malaria parasites in continuous culture. Science; 193: 673-675.

Tufet-Bayona M.; Janse C.J.; Khan S.M.; Waters A.P.; Sinden R.E.; Franke-Fayard B. (2009): Localisation and timing of expression of putative Plasmodium berghei rhoptry proteins in merozoites and sporozoites. Mol. Biochem. Parasitol.; 166: $22-31$

Van Dooren G.G.; Walker R.F.; Joiner K.A.; Roos D.S.; McFadden G.I. (2000): Traffic jams: protein transport in Plasmodium falciparum. Parasitol. Today; 16: 421-427.
Kats L.M.; Cooke B.M.; Coppel R.L.; Black C.G. (2008): Protein trafficking to apical organelles of malaria parasites building an invasion machine. Traffic; 9: 176-186.

Lambros C. and Vanderberg J.P.; (1979): Synchronization of Plasmodium falciparum erythrocytic stages in culture. J. Parasitol.; 65: 418-420.

Lee K.K.; Leung A.K.; Tang M.K.; Cai D.Q.; Schneider C.; Brancolini C.; Chow P.H. (2001): Functions of the growth

Yu Y.A.; Szalay A.A.; Wang G.; Oberg K. (2003): Visualization of molecular and cellular events with green fluorescent proteins in developing embryos: a review. Luminescence; 18: 1-18. 
Ahmed M. Ghoneim., Recombinant expression of the rhoptry protein Clag3.1 as a green fluorescent chimeric protein in Plasmodium falciparum.

(A) Gateway BP recon bination reaction:

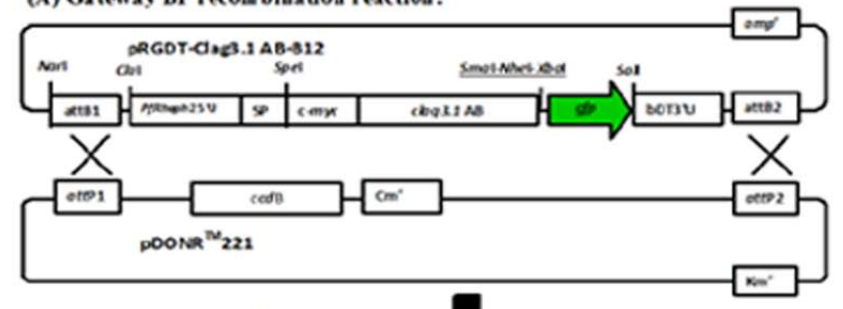

(B) Gateway LR recombiaation reactioa:

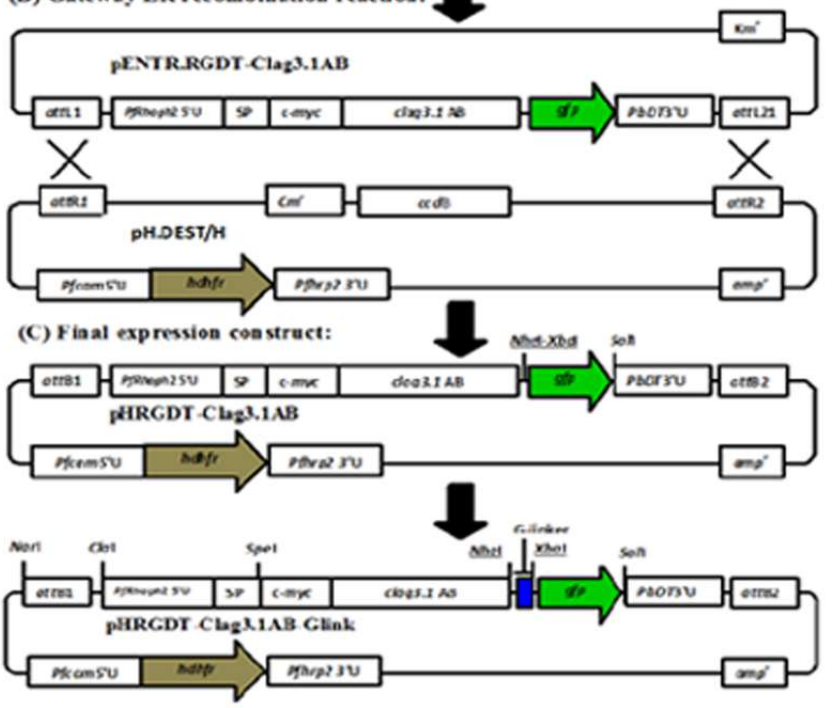

Big. 1: A schematic diagram illustrating how the transfection construct pHRGDT-Clag3.1AB was produced. The plasmid vector pRGDT-Clag3 . 1AB-B12 was developed from the plasmid pRGDT-Clag3. 1A-B12 (Ghoneim et al., 2007) by inserting a DNA fragment encoding the amino acids 461-1030 of Clag3.1 into the BgIII site that exists at $3^{\prime}$ end of clag3.1. (A) The attB expression clone (pRGDT-Clag3.1A-B12) was subjected to BP Gateway recombination reaction with the donor vector pDONR ${ }^{\mathrm{m}} 221$ to produce the entry clone pENTR.RGDT-Clag3.1AB with attL recombination sites (B), which was then subjected to LR recombination reaction with the destination vector pH.DEST/H containing the attR recombination sites (Ghoneim et al., 2007) to produce the transfection construct pH.RGDT-Clag3.1AB (C), into which the Glycine-rich linker encoding sequence was ligated to produce the final construct pH.RGDT-Clag3.1AB-Glink. 
(A) Restriction digestion of plasmid vectors
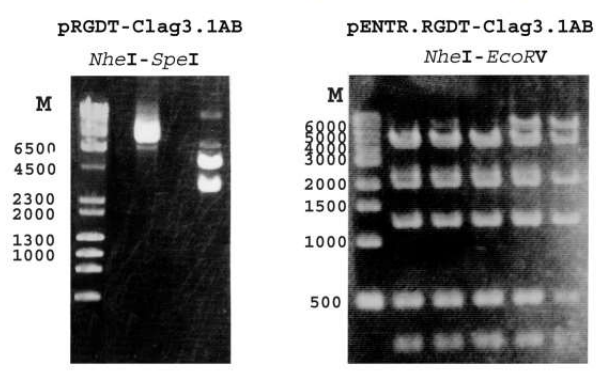

PH. RGDT-Clag3.1AB

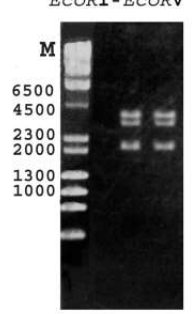

(B) Synchronized parasite culture

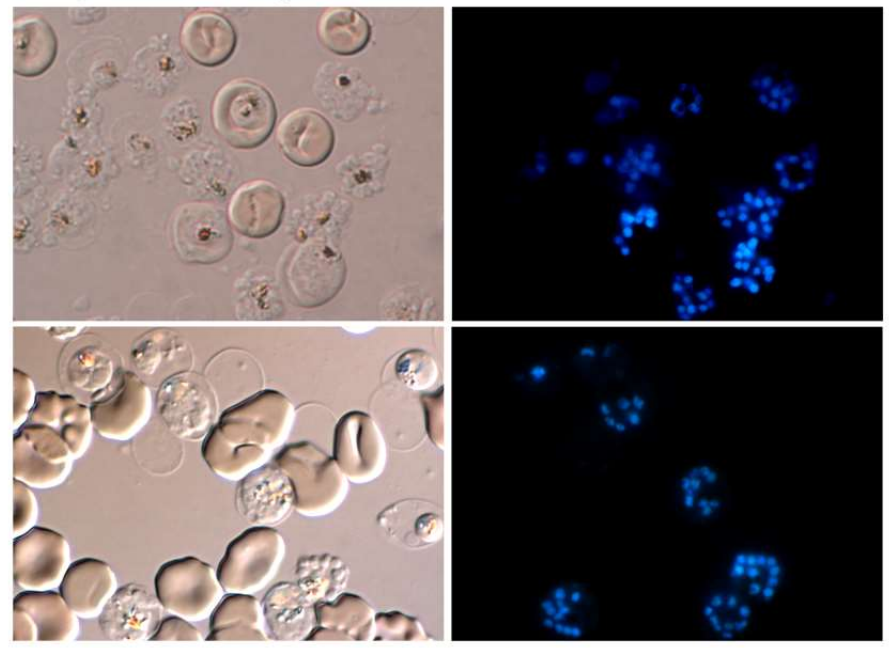

Fig. 2: (A) Confirmation of the plasmid size by restriction digestion of the vectors produced in this study. Digestion of pRGDT-Clag3.1AB-B12 with NheI and SpeI resulted in the expected size-products, 3160 bp and 5255 bp. Undigested plasmid was loaded along with a digested one. Digestion of pENTR.RGDT-Clag3.1AB with NheI and EcoRv resulted in the fragments $4367 \mathrm{bp}, 2004 \mathrm{bp}, 1259 \mathrm{bp}, 491$ and $266 \mathrm{bp}$ as expected. Five plasmid clones of digested plasmids were loaded onto the gels. Digestion of pH.RGDT-Clag3.1AB with ECORI and ECORV resulted in the expected size-products; 3656 bp, $3098 \mathrm{bp}, 1995 \mathrm{bp}$ and $1972 \mathrm{bp}$. (B) Representative images of schizont-stage $P$. falciparum culture. Left column contains DIC images of live parasites. Right column shows the same parasite culture stained with the nucleus-specific stain DAPI. 
(A) (1) Clag3.1

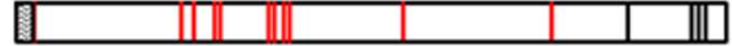

(ii) RhopH2-Myc-Clag3 $1_{(24-1064)}$-GPP

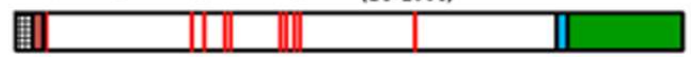

(B) Sequence of RhopH2-Myc-Clag3 ${ }^{1} 1_{(24-1064)}-$ GFP

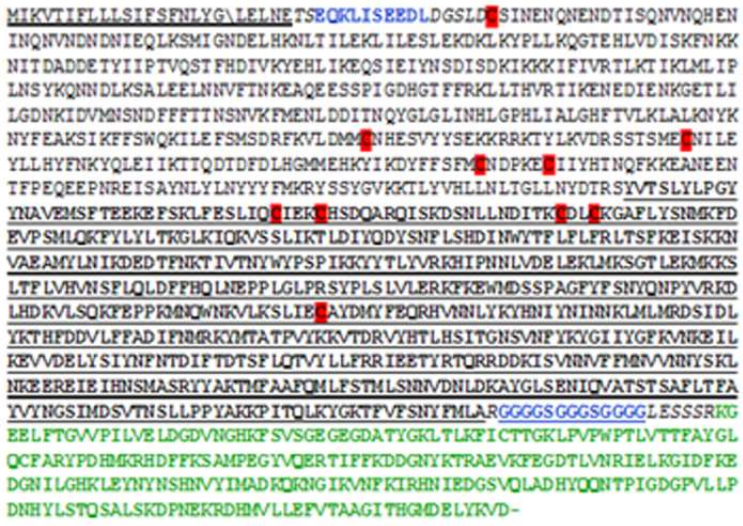

Fig. 3: (A) Schematic representation for the domain structure of the endogenous Clag3.1 and RhopH2-MycClag3.1(24-1064)-GFP chimeric protein expressed in transgenic Plasmodium falciparum. (i) A schematic diagram of the domain structure of the endogenous Clag3.1 with its signal peptide (zigzag box). (ii) RhopH2-MYc-Clag3.1(24-1064)-GFP chimeric protein expressed from the construct pH.RGDT-Clag3.1AB-Glink. This protein contains the putative signal peptide sequence of PfRhopH2 (stripped box) and c-Myc tag (brown box) at its $\mathrm{N}$-terminus. Red vertical lines indicate Cysteine residues. Blue box indicates the linker region and green box represents GFP (B) Amino acid sequence of the RhopH2-MYc-Clag3.1(24-1064)-GFP chimeric protein. Underlined residues are the first $N-$ terminal 24 amino acid from RhopH2 with the signal peptide cleavage site indicated with a back slash, normal blue residues represent $c-M y c$ tag, conserved cysteine residues are shown in red, normal black residues are the N-terminus of Clag3.1 (after the signal peptide), black underlined residues represent the middle $1 / 3$ of Clag3.1, underlined blue residues represent the glycine-rich linker, black residues around the linker were introduced for cloning purposes and green bold residues represent GFP. 
(A)

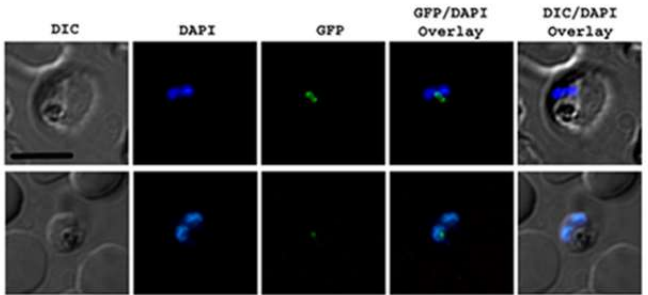

(B)

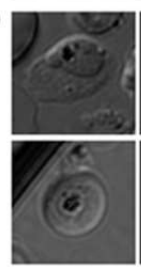

(C)

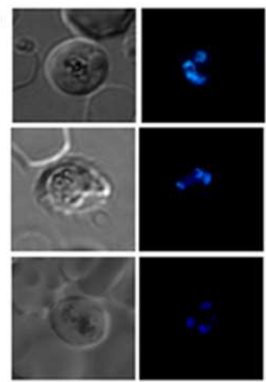

(D)

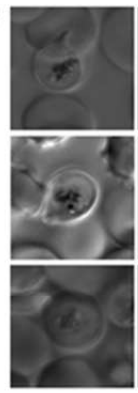

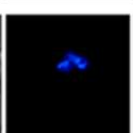
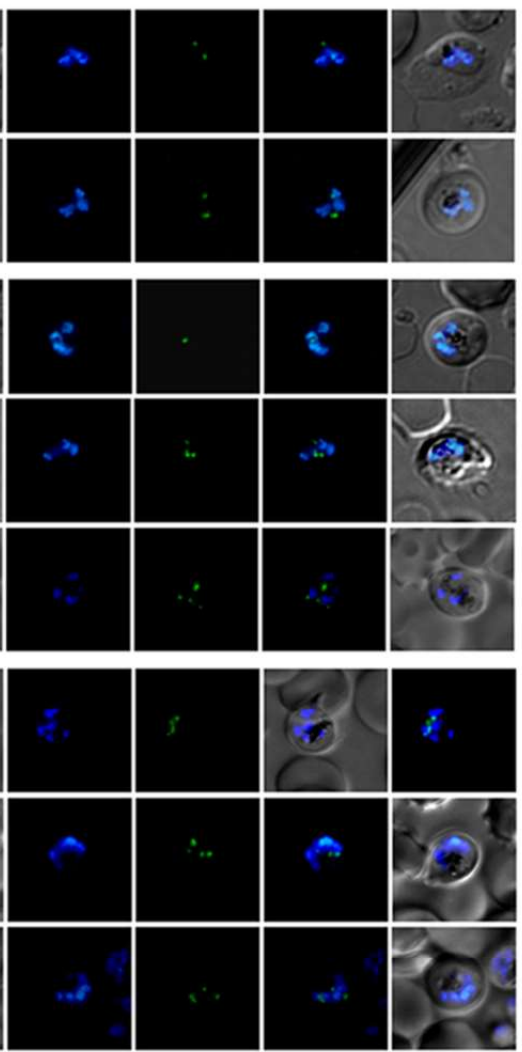

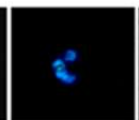

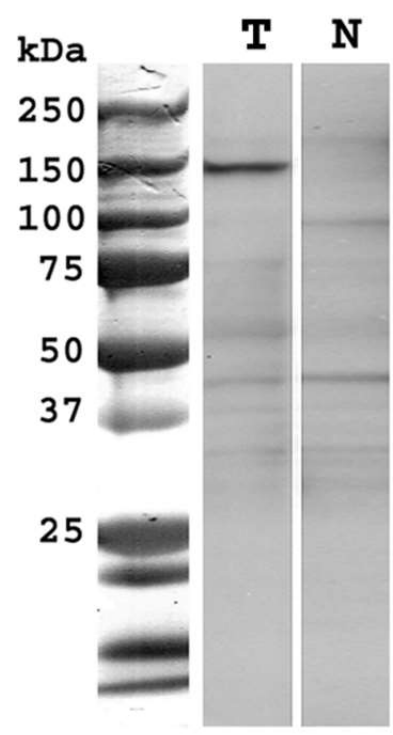

Fig. 5: Western blot Analysis of the expressed RhopH2-MycClag3.1 (24-1064) -GFP chimeric protein. Proteins were extracted from the transfected parasites (T) and nontransfected parasites (N) and stained with anti-C-Myc. A specific band close to $150 \mathrm{kDa}$ was detected and thus confirms the expression of integral RhopH2-MYc-

Fig. 4: Tracking the expression of the chimeric protein RhopH2Myc-Clag3.1(24-1064)-GFP in the late stages of transgenic Plasmodium falciparum parasites. A: Two nuclei-stage schizont. B: Three nuclei-stage schizont C: Four nuclei-stage schizont. D: Five nuclei-stage schizont. This indicates the successful expression of GFP after the relatively large Clag3.1 and thus validating the rhoph2 promoter to drive the expression of large chimeric proteins. In all cases, the GFP chimera was retained in a specific compartment; most likely endoplasmic reticulum or Golgi of the parasite. Scale bar $=5 \mu$. 